\title{
Симультанная торакоскопическая
}

\section{пластика диафрагмы с радиочастотной абляцией левого предсердия и резекцией его ушка}

\author{
Базаров Д.В. ${ }^{1}$, Быстров Д.0. ${ }^{2}$, Шонбин А.Н. ${ }^{2}$, Казарян Г.А. ${ }^{1}$ \\ ${ }^{1}$ Государственный научный центр Российской Федерации Федеральное государственное бюджетное научное \\ учреждение «Российский научный центр хирургии имени академика Б.В. Петровского», 119991, г. Москва, Рос- \\ сийская Федерация \\ 2 Государственное бюджетное учреждение здравоохранения Архангельской области «Первая городская клиниче- \\ ская больница им. Е.Е. Волосевич», 163001, г. Архангельск, Российская Федерация
}

Описан первый в России успешный опыт симультанного подхода в хирургическом лечении релаксации правого купола диафрагмы и рецидива фибрилляции предсердий. Обсуждены технические аспекты оперативного вмешательства и перспективы развития минимально инвазивной хирургии в торакальной и сердечно-сосудистой хирургии.

Финансирование. Работа выполнена в рамках государственного задания ГНЦ РФ ФГБНУ РНЦХ им. акад. Б.В. Петровского (№ 0394-2021-0004)

Конфликт интересов. Авторы заявляют об отсутствии конфликта интересов.

Для цитирования: Базаров Д.В., Быстров Д.О., Шонбин А.Н., Казарян Г.А. Симультанная торакоскопическая пластика диафрагмы с радиочастотной абляцией левого предсердия и резекцией его ушка // Клиническая и экспериментальная хирургия. Журнал имени академика Б.В. Петровского. 2021. Т. 9, № 2. С. 131-135. D0I: https://doi.org/10.33029/23081198-2021-9-2-131-135

Статья поступила в редакцию 21.04.2021. Принята в печать 15.05.2021.

Simultaneous VATS diaphragm plication with radiofrequency ablation and auricle resection of the left atrium

Bazarov D.V. ${ }^{1}$, Bystrov D.0. ${ }^{2}$, Shonbin A.N. ${ }^{2}$, Kazaryan G.A. ${ }^{1}$

${ }^{1}$ Petrovsky National Research Center of Surgery, 119991, Moscow, Russian Federation

${ }^{2}$ City Hospital \#1 named after E.E. Volosevitch, 163001, Arkhangelsk, Russian Federation

The first successful experience of simultaneous approach in surgical treatment of the diaphragm relaxation and relapse of atrial fibrillation is described. The technical aspects of surgical intervention and the prospects for the development of minimally invasive surgery in thoracic and cardiovascular surgery are discussed.

Funding. The work was performed within the framework of a state assignment Petrovsky National Research Center of Surgery (No. 0394-2021-0004)

Conflict of interest. The authors declare no conflict of interest.

For citation: Bazarov D.V., Bystrov D.0., Shonbin A.N., Kazaryan G.A. Simultaneous VATS diaphragm plication with radiofrequency ablation and auricle resection of the left atrium. Clinical and Experimental Surgery. Petrovsky Journal. 2021; 9 (2): 131-5. DOI: https://doi.org/10.33029/2308-1198-2021-9-2-131-135 (in Russian)

Received 21.04.2021. Accepted 15.05.2021.

\section{ДЛЯ КОРРЕСПОНДЕНЦИИ}

Казарян Григорий Александрович младший научный сотрудник отделения торакальной хирургии и онкологии ГНЦ РФ ФГБНУ «РНЦХ им. акад. Б.В. Петровского» (Москва, Российская Федерация) E-mail: grigoriykazar@gmail.com https://orcid.org/0000-0002-05459078

\section{Ключевые слова:} видеоассистированная торакоскопическая хирургия, релаксация диафрагмы, торакоскопическая пластика диафрагмы, радиочастотная абляция, фибрилляция предсердий

CORRESPONDENCE

Grigoriy A. Kazaryan - Junior Researcher of the Department of Thoracic Surgery and Oncology, Petrovsky National Research Center of Surgery (Moscow, Russian Federation)

E-mail: grigoriykazar@gmail.com https://orcid.org/0000-0002-05459078

Keywords:

video-assisted thoracoscopic surgery (VATS), diaphragm palsy, VATS diaphragm plication, radiofrequency ablation, atrial fibrillation 
$\Phi$ ибрилляция предсердий (ФП) - наиболее частая форма нарушения ритма сердца, при водящая к увеличению рисков развития хронической сердечной недостаточности, инсультов, а также к общей смертности [1]. ФП возникает в результате поступления множества импульсов из автономных очагов, которые чаще всего расположены в устьях легочных вен. В 1998 г. М. Haїssaguerre и соавт. доказали, что эктопическая активность в легочных венах может являться триггером ФП [2]. Данное открытие, а также недостаточная эффективность медикаментозной терапии послужили толчком для развития катетерных методик лечения ФП. Согласно современным представлениям, катетерная абляция (КА) рекомендована пациентам с симптоматической пароксизмальной формой ФП, резистентной к консервативной терапии [3].

Одним из нечастых осложнений катетерной абляции является повреждение диафрагмального нерва, что связано с термическим воздействием во время проведения процедуры [4]. Правая сторона повреждается чаще благодаря близкому располо-

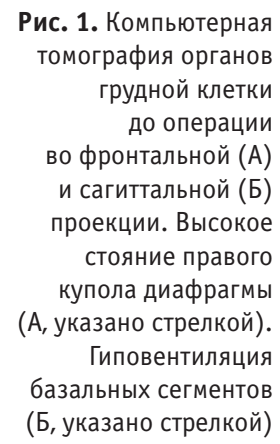

Fig. 1. Computer tomography of the chest organs before surgery in the frontal $(A)$ and sagittal (B) projections.

The high position

of the right dome

of the diaphragm in Fig. A

(indicated by an arrow).

Hypoventilation of the basal segments in Fig. B (indicated by an arrow)

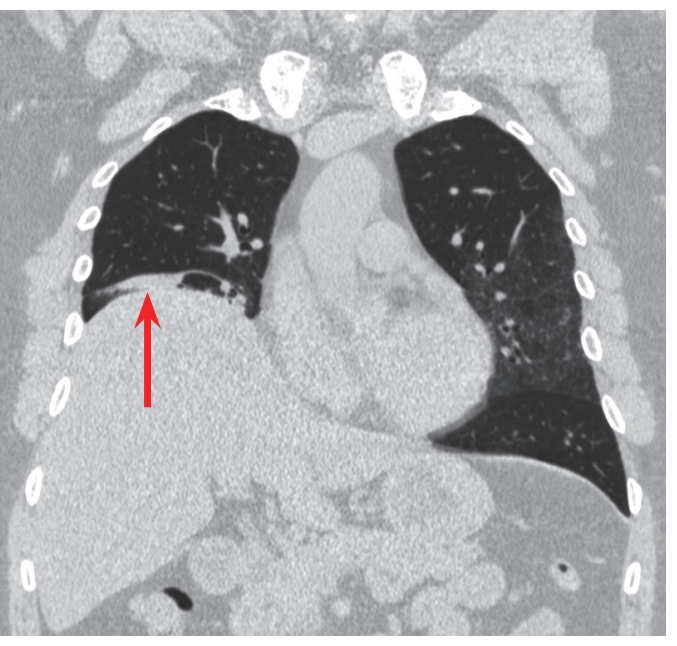

$A(A)$

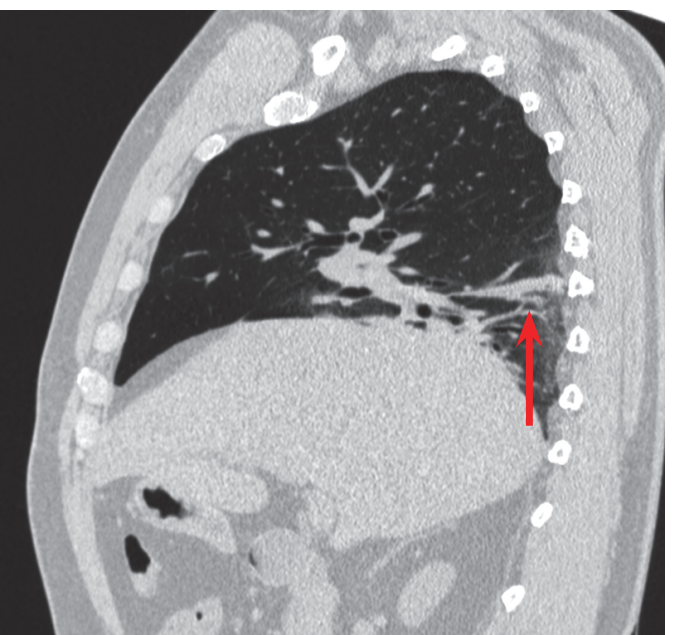

Б (B) жению диафрагмального нерва к верхней легочной и верхней полой вене [5]. Неполная изоляция легочных вен приводит к развитию рецидива ФП.

В доступной медицинской литературе мы не встретили описания подобной хирургической тактики при сочетании релаксации диафрагмы и рецидива ФП, поэтому приводим собственное наблюдение. На наш взгляд, наиболее оправданный и безопасный вариант лечения пациентов с релаксацией диафрагмы и рецидивом ФП - выполнение симультанной операции: пластики диафрагмы и радиочастотной абляции левого предсердия, (ЛП) которая позволяет одномоментно скорректировать оба патологических процесса.

\section{Клинический случай}

Пациент К., 53 года, госпитализирован в отделение кардиохирургии с клинико-рентгенологической картиной релаксации правого купола диафрагмы и рецидива ФП.

С 2015 г. у пациента регистрировались пароксизмы ФП, а с 2017 г. - персистирующая форма, по поводу которой проводили антиаритмическую и антикоагулянтную терапию, без значимого эффекта. В мае 2018 г. пациенту выполнена катетерная криоабляция устьев легочных вен, на 6-е сутки после вмешательства зафиксирован рецидив ФП, купированный консервативно. После выписки из стационара пациент стал предъявлять жалобы на выраженную одышку (EHRAIII, MCA 3), снижение толерантности к физическим нагрузкам. Пациенту проводили консервативное лечение без должного положительного эффекта. В ходе проведенного обследования (см. таблицу) на компьютерной томографии органов грудной клетки выявлены высокое стояние правого купола диафрагмы, компрессионный ателектаз нижней и средней долей правого легкого (рис. 1, А, Б), также по данным холтеровского мониторирования ЭКГ сохранялись персистирующая форма ФП и выраженное снижение показателей вентиляционной функции легких на спирометрии. Принято решение о проведении симультанной операции в объеме торакоскопической пластики правого купола диафрагмы и радиочастотной абляции (РЧА) ЛП с резекцией ушка.

\section{Техника операции}

I этап: торакопорт 10 мм установлен в третьем межреберье по средней подмышечной линии справа (рис. 2), купол диафрагмы располагался на уровне третьего-четвертого межреберья. Инсуффляция $\mathrm{CO}_{2}$ в правую плевральную полость. Дополнительные порты установлены в седьмом и восьмом межреберьях по передней и задней подмышечным линиям. Формировалась дупликатура купола диа- 


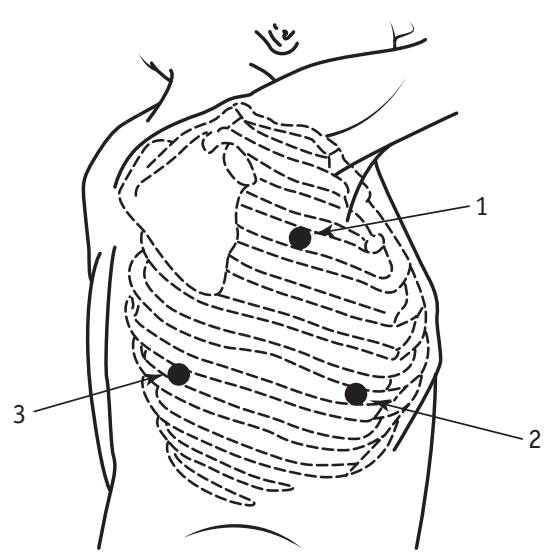

Рис. 2. Расположение торакопортов при пластике правого купола диафрагмы. 1-й порт - третье межреберье по средней подмышечной линии, 2-й порт - седьмое межреберье по передней подмышечной линии, 3-й порт восьмое межреберье по задней подмышечной линии

Fig. 2. Thoracoports placement during diaphragmatic plication. 1 port - 3 intercostal space along the middle axillary line, 2 port -7 intercostal space along the anterior axillary line, 3 port -8 intercostal space along the posterior axillary line

фрагмы в направлении спереди назад (дупликатура фиксировалась П-образными синтетическими швами на фетровых прокладках).

II этап проводился с использованием радиочастотного генератора AtriCure.

Справа были использованы торакопорты, через которые осуществлялась пластика диафрагмы, дополнительный порт был установлен в шестом межреберье по задней подмышечной линии (рис. 3). Проводилась РЧА-изоляция правых легочных вен (14 аппликаций до достижения трансмуральности) биполярным зажимом AtriCure. Формировалась Roof Lesion Line и Bottom Lesion Line монополярным электродом MLP AtriCure (по 6 аппликаций до достижения трансмуральности).

Слева установка торакопортов была идентичной. Выполнялась РчА-изоляция левых легочных вен (14 аппликаций до достижения трансмуральности). Формировалась линия у основания ушка левого предсердия Auriculum Line монополярным электродом MLP AtriCure (по 6 аппликаций до достижения трансмуральности). Ушко ЛП прошивалось и резецировалось эндоскопическим сшивающим аппаратом.

Продолжительность операции составила 5 ч 30 мин. Кровопотеря была минимальной.

Схема данного этапа операции представлена на рис. 4.

Сразу после проведения операции у пациента восстановился синусовый ритм, снизилась одышка. Послеоперационный период протекал без осложнений.

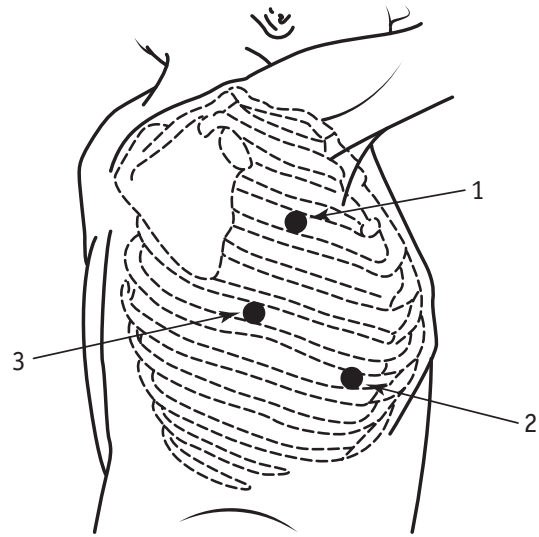

Рис. 3. Расположение торакопортов при радиочастотной абляции левого предсердия. 1-й порт - третье межреберье по средней подмышечной линии, 2-й порт - седьмое межреберье по передней подмышечной линии, 3-й порт шестое межреберье по задней подмышечной линии

Fig. 3. Thoracoports placement during radiofrequency ablation of the left atrium. 1 port - 3 intercostal space along the middle axillary line, 2 port - 7 intercostal space along the anterior axillary line, 3 port - 6 intercostal space along the posterior axillary line

Спустя 2 года после операции у пациента отсутствует одышка при любой физической нагрузке, нет нарушений ритма сердца. На компьютерной томографии через 24 мес после операции признаков релаксации правого купола диафрагмы нет (рис. 5). По данным контрольной спирометрии через 24 мес отмечается прирост показателей на $20 \%$ : FVC $-67 \%, \mathrm{FEV}_{1}-62 \%, \mathrm{FEV}_{1} / \mathrm{FVC}-77 \%$.

\section{Обсуждение}

Катетерная абляция является эффективным методом лечения пароксизмальной формы ФП. При данной процедуре отмечается низкий уровень осложнений и летальности, а рецидив заболевания встречается в 18-20\% случаях [5]. Среди общего числа осложнений КА следует выделить повреждение диафрагмального нерва в результате термического воздействия. По данным J. Anadre и соавт., частота встречаемости данного осложнения достигает 4,73\% [6].

Одними из первых релаксации диафрагмы через торакотомию выполнили J. Morley и соавт. в 1920 г. [7]. В России первым фундаментальным трудом стала монография «Хирургия диафрагмы», опубликованная в 1965 г. Б.В. Петровским и соавт. [9]. Она включала не только весь мировой опыт того времени, но и собственный опыт авторов, приобретенный при лечении почти 2000 больных с различной патологией диафрагмы, в том числе 42 пациентов с релаксацией. B 1990 г. D. Graham и соавт. [10] и в 2002 г. S. Higgs [11] опубликовали 
Данные инструментальных методов исследования пациента К.

\begin{tabular}{|c|c|}
\hline Метод исследования & Результат \\
\hline Коронарография от 24.03.2019 & Правый тип кровоснабжения сердца. Коронарные артерии без патологии \\
\hline Эхокардиография от 24.03.2019 & $\begin{array}{l}\text { ФВ ЛЖ - 63\%, КДР/КСР - 52/34 мм. ЛП - } 43 \text { мм. Давление в ЛА - } 28 \text { мм рт.ст. } \\
\text { Патологии клапанов нет. Зон нарушения сократимости не выявлено }\end{array}$ \\
\hline $\begin{array}{l}\text { 24-часовое холтеровское } \\
\text { мониторирование ЭКГ } \\
\text { от 24.03.2019 }\end{array}$ & $\begin{array}{l}\text { За время мониторирования (22 ч } 36 \text { мин) регистрировалась фибрилляция } \\
\text { предсердий с минимальной ЧСС - } 58 \text { в минуту }(6: 05-\text { «сон»), максимальной } \\
\text { чСС - } 107 \text { в минуту (12:58 - «ходьба»). Средняя ЧСС - } 90 \text { в минуту. }\end{array}$ \\
\hline Спирометрия от 24.03.2019 & $\begin{array}{l}\mathrm{FVC}-1,50 \text { л, } 47 \% . \mathrm{FEV}_{1}-1,09 \text { л, } 42 \% . \mathrm{FEV}_{1} / \mathrm{FVC}-57 \% . \text { MOC } 25-75 \% . \\
\text { ПОС - 28\%. Нарушения вентиляционной функции легких по смешанному } \\
\text { типу, значительное снижение ЖЕЛ }\end{array}$ \\
\hline
\end{tabular}

Примечание. ФВ ЛЖ - фракция выброса левого желудочка; КДР - конечно-диастолический размер; КСР - конечный систолический размер; ЛП - левое предсердие; ЛА - легочная артерия; ФП - фибрилляция предсердий; чСС - частота сердечных сокращений; FVC - форсированная жизненная емкость легких; FEV - объем форсированного выдоха за 1 с; MOC - мгновенные объемные скорости; ПОС - пиковая объемная скорость $28 \%$; ЖЕЛ - жизненная емкость легких.

Рис. 4. Схема торакоскопической абляции левого предсердия

Fig. 4. Illustration of the left atruim's thoracoscopic ablation

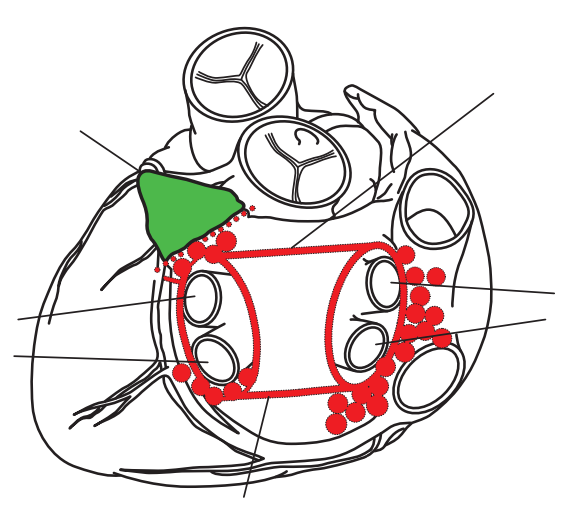

Рис. 5. Компьютерная томография органов грудной клетки (через 24 мес после операции). Правый купол диафрагмы расположен на уровне VIII ребра. Ателектазов долей правого легкого нет

Fig. 5. Chest CT scan (24 months after surgery). The right dome of the diaphragm is located at the level of 8th rib. There are no atelectases of the right lung

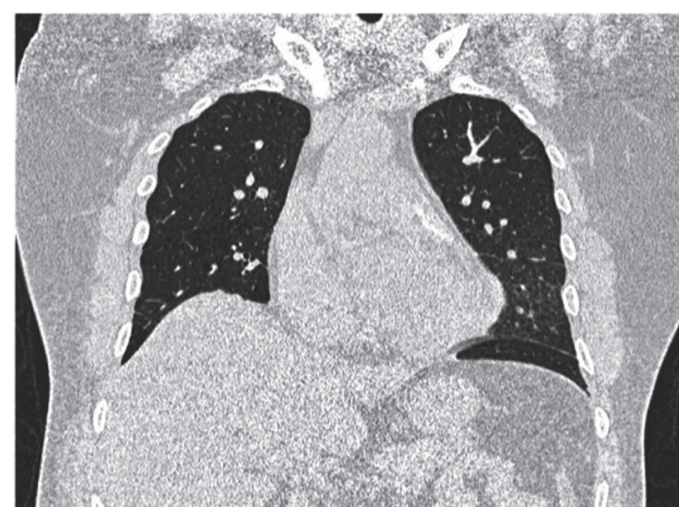

5- и 10-летние результаты операций по методике пликации (формирование дупликатуры с использованием П-образных швов на тефлоновых прокладках) диафрагмы. Данный вид вмешательства доказал свою эффективность и надежность.

С 2000-х гг. благодаря развитию видеоэндоскопических технологий стали внедряться минимально инвазивные методики пластики диафрагмы. R. Freeman первым продемонстрировал преимущество видеоторакоскопической пластики диафрагмы. Отмечалось улучшение показателей функции внешнего дыхания, уменьшение одышки и общего количества койко-дней $[12,13]$.
В настоящее время данное вмешательство можно проводить, используя однопортовый доступ, как и было выполнено в другом нашем наблюдении [14]. Это позволяет уменьшить операционную травму, снизить частоту послеоперационных осложнений, улучшить эстетический результат операции.

Мировой опыт лечения пациентов с релаксацией диафрагмы невелик. Отчасти это связано с бессимптомным течением патологии, и в таких случаях проведение хирургического вмешательства не обосновано. Доказано, что проведение хирургической коррекции односторонней релаксации диафрагмы показано лишь при наличии клинических проявлений данной патологии.

При обзоре зарубежной и отечественной литературы мы не встретили публикаций о симультанной торакоскопической пластике диафрагмы и РЧА ЛП. В данном случае такая тактика вмешательства была выбрана в связи с наличием у пациента дыхательной недостаточности и затрудненного доступа к ЛП на фоне релаксации правого купола диафрагмы. Для проведения симультанных операций требуется не только высококвалифицированная работа всей операционной, но и достаточные ресурсы клиники для обеспечения безопасности пациента в периоперационном периоде. В связи с этим подобные операции рекомендуется проводить в специализированных стационарах.

\section{Заключение}

Данный клинический случай демонстрирует успешное применение симультанного подхода к хирургической коррекции осложнения в виде релаксации правого купола диафрагмы и выполнения повторной процедуры устранения ФП с применением торакоскопических технологий. На наш взгляд, использование такого подхода является оптимальным методом лечения пациентов с ФП и релаксацией диафрагмы и сопровождается хорошими непосредственными и отдаленными результатами. 
https://www.youtube.com/watch?v=EyF8_6mfqBY\&t=2s

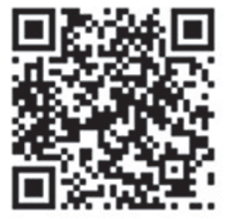

Сканируя данный QR-код, вы имеете возможность посмотреть видеозапись операции.

\section{Литература}

1. Bosch N.A., Cimini J., Walkey A.J. Atrial fibrillation in the ICU // Chest. 2018. Vol. 154, N 6. P. 1424-1434.

2. Haïssaguerre M., Jaïs P., Shah D.C. et al. Spontaneous initiation of atrial fibrillation by ectopic beats originating in the pulmonary veins // N. Engl. J. Med. 1998. Vol. 339. P. 659-666.

3. January C.T. et al. 2019 AHA/ACC/HRS focused update of the 2014 AHA/ACC/HRS guideline for the management of patients with atrial fibrillation: a report of the American College of Cardiology/American Heart Association Task Force on Clinical Practice Guidelines and the Heart Rhythm Society // J. Am. Coll. Cardiol. 2019. Vol. 74, N 1. P. 104-132.

4. Sacher F. et al. Phrenic nerve injury after atrial fibrillation catheter ablation: characterization and outcome in a multicenter study // J. Am. Coll. Cardiol. 2006. Vol. 47, N 12. P. 2498-2503.

5. Marrouche N.F. et al. Catheter ablation for atria fibrillation with heart failure // N. Engl. J. Med. 2018. Vol. 378, N 5. P. 417-427.

6. Casado-Arroyo R. et al. Phrenic nerve paralysis during cryoballoon ablation for atrial fibrillation: a comparison between the first-and second-generation balloon // Heart Rhythm. 2013. Vol. 10, N 9. P. 1318-1324.

7. Andrade J.G. et al. Efficacy and safety of cryobalIoon ablation for atrial fibrillation: a systematic review of published studies // Heart Rhythm. 2011. Vol. 8, N 9. P. 1444-1451.

\section{References}

1. Bosch N.A., Cimini J., Walkey A.J. Atrial fibrillation in the ICU. Chest. 2018; 154 (6): 1424-34.

2. Haïssaguerre M., Jaïs P., Shah D.C., et al. Spontaneous initiation of atrial fibrillation by ectopic beats originating in the pulmonary veins. N Engl J Med. 1998; 339: 659-66.

3. January C.T., et al. 2019 AHA/ACC/HRS focused update of the 2014 AHA/ACC/HRS guideline for the management of patients with atrial fibrillation: a report of the American College of Cardiology/American Heart Association Task Force on Clinical Practice Guidelines and the Heart Rhythm Society. J Am Coll Cardiol. 2019; 74 (1): 104-32.

4. Sacher F., et al. Phrenic nerve injury after atria fibrillation catheter ablation: characterization and outcome in a multicenter study. J Am Coll Cardiol. 2006; 47 (12): 2498-503.

5. Marrouche N.F., et al. Catheter ablation for atrial fibrillation with heart failure. N Engl J Med. 2018; 378 (5): 417-27.

6. Casado-Arroyo R., et al. Phrenic nerve paralysis during cryoballoon ablation for atrial fibrillation: a comparison between the first-and second-generation balloon. Heart Rhythm. 2013; 10 (9): 1318-24.

7. Andrade J.G., et al. Efficacy and safety of cryoballoon ablation for atrial fibrillation: a systematic review of published studies. Heart Rhythm. 2011; 8 (9): 1444-51.
8. Panda N., Cheung J. Complications from catheter ablation of atrial fibrillation: impact of current and emerging ablation technologies // Curr. Treat. Options Cardiovasc. Med. 2014. Vol. 16, N 10. P. 344.

9. Петровский Б.В., Каншин Н.Н., Николаев Н.О Хирургия Аиафрагмы. Москва : Медицина, 1965. С. 244-283.

10. Diaphragmatic plication for unilateral diaphragmatic paralysis: a 10-year experience // Ann. Thorac. Surg. 1990. Vol. 49. P. 248-252.

11. Higgs S. et al. Long term results of diaphragmatic plication for unilateral diaphragm paralysis // Eur. J. Cardiothorac. Surg. 2002. Vol. 21. P. 294-297.

12. Freeman R.K., Wozniak T.C., Fitzgerald E.B. Functional and physiologic results of video-assisted thoracoscopic diaphragm plication in adult patients with unilateral diaphragm paralysis // Ann. Thorac. Surg. 2006. Vol. 81. P. 1853-1857.

13. Freeman R., Van Woerkom J., Vyverberg A., Ascioti A. Long-term follow-up of the functional and physiologic results of diaphragm plication in adults with unilateral diaphragm paralysis // Ann. Thorac. Surg. 2009. Vol. 88. P. 1112-1117.

14. Григорчук А.Ю., Базаров А.В., Выжигина М.А. Кавочкин А.А., Кабаков А.Г. Хирургия релаксации Аиафрагмы: со времен Б.В. Петровскогодо нашихАней// Хирургия. Журнал имени Н.И. Пирогова. 2018. № 7. C. 60-66. DOI: https://doi.org/10.17116/hirurgia2018760

8. Panda N., Cheung J. Complications from catheter ablation of atrial fibrillation: impact of current and emerging ablation technologies. Curr Treat Options Cardiovasc Med. 2014; 16 (10): 344.

9. Petrovsky B.V., Kanshin N.N., Nikolaev N.O. Diaphragm surgery. Moscow: Meditsina, 1965: 244-3. (in Russian)

10. Diaphragmatic plication for unilateral diaphrag matic paralysis: a 10-year experience. Ann Thorac Surg. 1990; 49: 248-52.

11. Higgs S., et al. Long term results of diaphrag matic plication for unilateral diaphragm paralysis. Eur J Cardiothorac Surg. 2002; 21: 294-7.

12. Freeman R.K., Wozniak T.C., Fitzgerald E.B. Functional and physiologic results of video-assisted thoracoscopic diaphragm plication in adult patients with unilateral diaphragm paralysis. Ann Thorac Surg. 2006; 81: $1853-7$.

13. Freeman R., Van Woerkom J., Vyverberg A., Ascioti A. Long-term follow-up of the functional and physiologic results of diaphragm plication in adults with unilateral diaphragm paralysis. Ann Thorac Surg. 2009; 88: 1112-7.

14. Grigorchuk A.Yu., Evseev E.P., Vyzhigina M.A., Kavochkin A.A., Kabakov D.G. Diaphragm relaxation surgery: since B.V. Petrovsky to the present day. Khirurgiya. Zhurnal imeni N.I. Pirogova. [Surgery. The Journal Named after N.I. Pirogov]. 2018; (7): 60-6. DOI: https://doi. org/10.17116/hirurgia2018760 (in Russian) 